\author{
IZABELA ORCHOWSKA \\ Uniwersytet im. Adama Mickiewicza w Poznaniu \\ izaorch@amu.edu.pl
}

\title{
Dimension discursive de la construction et de la diffusion des savoirs sur la formation des enseignants de LE au sein de la communauté glottodidactique polonaise
}

\author{
Discursive dimension of the construction \\ and the circulation of knowledge connected with \\ the issue of foreign language teacher education \\ within the Polish glottodidactic community
}

\begin{abstract}
The paper concerns the discursive dimension of the construction and the circulation of knowledge connected with the issue of foreign language teacher education. A starting point for the discussion is the assumption according to which glottodidactics as a science is still perceived in a highly heterogenic way by members of the Polish glottodidactic community. Not only is the subject of glottodidactics so complex and interdisciplinary that the multitude of its possible interpretations does not allow us to decide on one common epistemological paradigm, but glottodidactic discourse is also very heterogeneous. The author of this article presents the conclusions from a metaglottodidactic analysis of articles covering the subject of foreign language teacher training which were published in Neofilolog in 1990-2010, and answers the question of whether, and with what kind of discourse configurations do Polish glottodidacticians theoretically locate their propositions for educating teachers presented in their publications.
\end{abstract}

KEYWORDS: discursive dimension of the construction and the circulation of scientific knowledge, epistemological specificity of glottodidactics, theoretical stance of glottodidactic discourse, metaglottodidactic research, scientific dialogue.

MOTS-CLES : dimension discursive de la construction et de la diffusion des savoirs scientifiques, spécificité épistémologique de la glottodidactique, cadrage théorique du discours glottodidactique, recherche méta-glottodidactique, dialogue scientifique. 


\section{INTRODUCTION}

Le présent article sera consacré à la problématique de la construction et de la diffusion des savoirs sur la formation des enseignants de langues étrangères dans et à travers le discours glottodidactique transmis en polonais ou en anglais dont les auteurs sont membres de la commuanuté glottodidactique polonaise. Notre réflexion sera donc située dans la catégorie des recherches méta-glottodidactiques qui portent sur "la science en train de se faire " (Lefebvre 2006), voire " en train de se dire " (Mondada 1995). Plus précisément, le discours scientifique dans le domaine de la glottodidactique constituera l'objet de notre méta-analyse et nous nous concentrerons sur les aspects épistémologiques de cet objet, en tentant de répondre à la question suivante : est-ce que - et si oui, par quels types de configurations discursives - les auteurs des articles publiés dans la revue Neofilolog définissent leur positionnement théorique et/ou le cadrage théorique de leurs réflexions portant sur la formation des enseignants de langues étrangères ?

Il est à noter, également à titre introductif, que nous comprenons le discours glottodidactique comme un discours de recherche et que nous le traitons comme un type particulier de discours spécialisé, à savoir le discours partagé par les membres de la communauté des spécialistes qui se différencient épistémologiquement des autres spécialistes par le fait qu'ils sont censés, premièrement, contribuer à construire et à diffuser les savoirs scientifiques dans leur domaine, et deuxièmement, proposer des applications de ces savoirs afin d'améliorer le processus d'enseignement/apprentissage des langues étrangères et les processus liés à celui-ci, y compris le processus de formation des enseignants de langues.

Quant au savoir portant sur la formation des enseignants de langues étrangères dont nous envisageons d'analyser dans le discours des spécialistes polonais en glottodidactique la construction et la diffusion, nous lui attribuons le statut de savoir spécialisé qui a un caractère hétérogène aussi bien au niveau des aspects thématiques auxquels il se rapporte (Orchowska 2015b) que sur le plan discursif relatif à la façon dont il est diffusé à travers le discours. De même sur le plan épistémologique et donc pour ce qui est de l'inscription du discours glottodidactique dans les savoirs antérieurs du domaine de référence ou bien dans les savoirs de disciplines connexes, il n'y a pas de modèle unique qui serve de référence à tous les scripteurs. De plus, les critères de scientificité appliqués lors de la construction de ce savoir diffèrent d'un spécialiste à l'autre, ce qui influence notamment les méthodologies de recherche qu'ils appliquent.

On se focalisera ici sur la dimension discursive du savoir glottodidactique, mais en raison du fait qu'il s'agit d'un savoir scientifique, construit et 
diffusé dans et par le discours de recherche, nous ne pouvons pas sousestimer, dans notre méta-réflexion, la dimension épistémologique du fonctionnement de ce savoir au sein de la communauté glottodidactique polonaise. En même temps, si l'on se penche sur la construction et la diffusion $\mathrm{du}$ savoir glottodidactique, il faut tenir compte du fait que ce savoir fait partie intégrale de la glottodidactique en tant que science et prendre en considération la spécificité épistémologique de cette science. Premièrement, nous devons admettre que l'appartenance de la glottodidactique aux sciences humaines (et sociales) signifie, entre autres, la complexité de son objet de recherche, voire son interdisciplinarité (Wilczyńska 2010), et par conséquent le pluriparadigmatisme au niveau de sa définition et/ou de sa description ainsi que l'approche pluriparadigmatique dans l'interprétation des données de recherche. Deuxièmement, le caractère empirique de l'objet de recherche permet de considérer cette science comme une science empirique ; ainsi, à la différence des représentants des sciences formelles focalisés sur les axiomes, les spécialistes en glottodidactique doivent définir leur objet de recherche dans le temps et dans l'espace et donc tenir compte de l'importance du contexte socio-culturel aussi bien pour ce qui est de la définition de l'objet de leur recherche que pour ce qui est du choix de la méthodologie de recherche et de l'interprétation des données. De plus, en glottodidactique, les rapports entre les théories scientifiques et les théories appliquées se présentent de façon spécifique. Plus précisément, les spécialistes en glottodidactique sont obligés de construire avant tout des savoirs théoriques et ensuite des savoirs appliqués (Dakowska 2014), sans toutefois ignorer la demande sociale à l'égard de leur domaine de recherche.

Troisièmement, si l'on parle de l'autonomie scientifique de la glottodidactique, il faut être conscient qu'il s'agit d'une autonomie relative de ce domaine de recherche en train de définir non seulement ses relations avec les disciplines connexes mais aussi son identité propre. En fait, au sein de la communauté glottodidactique polonaise, on admet aujourd'hui des représentations différentes, voire contradictoires de ce qu'est la glottodidactique. Ainsi, pour certains spécialistes, la glottodidactique n'est qu'un domaine pratique qui sert à améliorer les méthodes d'enseignement des langues étrangères, pour d'autres, c'est une des composantes de la linguistique appliquée, mais de plus en plus nombreux sont ceux qui y voient une science à part entière de plus en plus autonome, avec un objet et des méthodes de recherche qui lui sont propres. D'ailleurs, en 2014, l'Association Polonaise des Néophilologues (pl. Polskie Towarzystwo Neofilologiczne), avec le soutien des milieux académiques impliqués dans l'enseignement/apprentissage des langues étrangères, a déposé auprès du Ministère de l'Enseignement Supérieur et de la Recherche sa demande de reconnaissance de la 
glottodidactique en tant que science autonome ; justifiant cela par la spécificité de la glottodidactique en ce qui concerne son objet et sa méthodologie de recherche, l'autonomie de la recherche en glottodidactique, les théories et les publications du domaine ainsi que les démarches de recherche de ses représentants. Les auteurs de la demande précisent également qu'il n'est pas justifié d'attribuer aux spécialistes en glottodidactique les grades et les titres en linguistique appliquée, car ils représentent un domaine de recherche à part (Jaroszewska 2014 : 53). Si la demande de reconnaissance est accordée, l'autonomie externe (institutionnelle) de la glottodidactique sera renforcée, et le dialogue entre les spécialistes sur l'autonomie interne de cette science en sera facilité.

\section{COMMUNAUTÉ GLOTTODIDACTIQUE POLONAISE EN TANT QUE COMMUNAUTÉ DE DISCOURS}

La communauté glottodidactique en tant que communauté de recherche peut être considérée comme une communauté de discours. Selon Swales (1990), la communauté discursive dans le contexte universitaire est un groupe socio-rhétorique qui possède les six caractéristiques suivantes :

- un but commun accepté par tous les membres de la communauté,

- des mécanismes d'interaction entre eux,

- des mécanismes de participation garantissant l'information et le retour d'information,

- l'utilisation et la possession d'un ou plusieurs genres,

- l'acquisition d'un lexique spécifique,

- un rapport équilibré entre novices et experts.

Tout en se focalisant sur le fonctionnement discursif d'une telle communauté, Swales sous-estime sa dimension épistémologique qui est mise en valeur par T. Kuhn (1962). Or, dans notre réflexion méta-glottodidactique (Orchowska 2015a), nous envisageons de prendre en considération aussi bien la dimension discursive que la dimension épistémologique du fonctionnement de la communauté de recherche. Comme dans notre publication antérieure (Orchowska 2013 : 190), nous présupposons que l'activité de recherche est un travail dont l'écriture constitue l'une des dimensions essentielles et nous postulons que l'analyse de discours glottodidactique nécessite le recours explicite aux configurations discursives des théories dans lesquelles l'écriture de recherche s'inscrit.

Les recherches visant l'analyse du discours scientifique sont nombreuses mais elles sont orientées avant tout sur l'analyse des éléments linguistiques $\mathrm{du}$ discours ou bien sur la structure des genres de discours scientifique. Ce 
dernier cas de figure est bien illustré par la publication de J. M. Swales (1990) qui a proposé le modèle IMRD (Introduction - Méthodes - Résultats Discussion) en tant que modèle de structure type de l'article scientifique $\mathrm{Si}$ ce modèle nous intéresse, c'est parce que nous analysons le cadrage théorique des articles scientifiques en glottodidactique, en considérant ceux-ci comme une variante de discours de recherche. D'autre part, même si dans la plupart des articles le cadrage théorique est annoncé dans les introductions, tous les articles glottodidactiques ne suivent pas ce modèle et s'avèrent hétérogènes également au niveau de leurs structures.

Quant aux recherches précédentes portant sur le cadrage théorique du discours glottodidactique, elles sont quasi inexistantes. On peut toutefois considérer l'étude de D. Wiśniewska (2013) comme un pas considérable vers une réflexion méta-glottodidactique ainsi orientée. En fait, cette étude sur les recherches-actions réalisées dans le domaine de la didactique de l'anglais langue étrangère constitue une méta-étude holistique visant à définir la spécificité des recherches-actions en ce qui concerne les théories de référence, les pratiques d'enseignement analysées et les traits discursifs des comptes rendus de ces recherches. Il convient de préciser que cette méta-étude, basée sur un corpus de 128 articles présentant les recherches-actions et publiés en anglais entre 1992 et 2012 dans des revues internationales à travers le monde, conclut qu'il ne serait pas possible à présent d'indiquer les traits discursifs stables et les structures identiques dans les articles en question, tellement les comptes rendus anglophones sur les recherchesactions varient d'un auteur à l'autre.

En ce qui concerne le contexte universitaire polonais, il faut évoquer également la publication d'A. Niżegorodcew (2009) dans laquelle celle-ci propose une analyse de 69 articles portant sur l'enseignement/apprentissage des langues étrangères, en se focalisant sur les rôles que les scripteurs s'attribuent dans leurs textes. L'auteure distingue les rôles suivants : celui de chercheur, celui de rapporteur sur les articles d'autres spécialistes du domaine, celui de théoricien, celui d'enseignant qui joue avec sa créativité professionnelle. Or, pour A. Niżegorodcew, cette diversité des rôles que cherchent à jouer à travers leur discours les spécialistes polonais dans l'enseignement/apprentissage des langues étrangères peut inspirer une réflexion approfondie dans le domaine, mais en même temps cette hétérogénéité rend la structure des articles glottodidactiques peu claire et par conséquent leur réception par les lecteurs devient difficile.

Parallèlement, sur le terrain de recherches francophones, F. Boch, F. Grossmann et F. Rinck (2009) ont proposé une étude méta-linguistique du cadrage théorique dans 40 articles du domaine de la linguistique. L'objectif de cette étude était de mettre au jour les pratiques scripturales d'auteurs 
d'articles de linguistique, afin de prendre conscience des procédés par lesquels les linguistes contribuent à la mise en circulation des discours dans leur champ scientifique. Les chercheurs précisent que - pour eux - la circulation des discours est une diffusion des théories selon le principe de renouvellement et que les idées des auteurs, les notions qu'ils définissent et les questions qu'ils posent constituent des objets discursifs à analyser. Plus précisément, il s'agit d'une reconstruction d'objets discursifs qui n'est jamais neutre et les énonciateurs ne peuvent pas s'exonérer de leur responsabilité sociale et épistémologique à l'égard des discours qu'ils font circuler.

On peut donc constater que les recherches sur la communuaté glottodidactique en tant que communauté de discours constituent un terrain d'études qui peut encore être exploité par les chercheurs. Et il en est de même pour ce qui est des recherches portant sur les questions méta-glottodidactiques telles que : l'identité de la glottodidatique en tant que domaine de recherche, ses perspectives de développement en tant que science, ses relations avec les disciplines connexes, les critères de scientificité de la recherche glottodidactique, etc. (Dakowska $2014: 21$ ).

\section{DIFFERENTES CONFIGURATIONS DISCURSIVES DU CADRAGE THÉORIQUE DANS LES ARTICLES GLOTTODIDACTIQUES POLONAIS}

Nous présenterons maintenant les résultats de notre méta-analyse, répondant ainsi à la question posée dans notre introduction, qui porte sur le cadre théorique du discours glottodidactique. Le corpus, objet de notre analyse, est constitué de 60 articles publiés dans la revue Neofilolog entre les années 1990 et 2010 (cf. annexe). Ces articles ont été sélectionnés selon deux critères : ils abordent la problématique de la formation des enseignants de langues étrangères et ils ont été publiés en polonais ou en anglais, ce qui garantit leur accès linguistique à tous les membres de la communauté glottodidactique polonaise.

Tout d'abord, il est à noter que nous avons distingué six types de configurations discursives relatives au cadrage théorique des articles analysés et que 17 articles sur 60 manquent de références aux théories scientifiques en tant que cadrage théorique. De plus, dans deux textes (Wilczyńska 1998 ; 1999), nous avons affaire à une prise de position épistémologique à caractère implicite. Passons maintenant aux détails de notre méta-analyse en commençant par la présentation de six configurations discursives selon le cadrage théorique des articles illustré par le tableau ci-dessous : 
Table 1. Six configurations discursives des article glottodidactiques selon leurs cadrages théoriques

\begin{tabular}{|l|}
\hline Les cadrages théoriques des articles glottodidactiques \\
\hline (1) cadrage théorique explicite avec le recours aux théories élaborées \\
dans le champ de la glottodidactique ou dans celui des sciences \\
connnexes avec l'adoption de la perspective épistémologique \\
propre à la glottodidactique ; par exemple, S. Adamczak-Kryszto- \\
fowicz (2006) justifie la nécessité de mener des recherches glotto- \\
didactiques sur la formation des enseignants de langue allemande \\
(et des interprètes-traducteurs) inscrites dans l'approche intercul- \\
turelle, en évoquant les publications glottodidactiques antérieures \\
concrètes à ce sujet et en indiquant le manque de recherches \\
empiriques portant sur l'évaluation des objectifs, des contenus, des \\
techniques et des matériaux glottodidactiques susceptibles de con- \\
tribuer au développement de la compétence interculturelle de \\
communication des étudiants;
\end{tabular}

(2) cadrage théorique explicite du discours glottodidactique quand il s'inscrit dans la perspective des sciences connexes; par exemple, J. Majer (2009) annonce dans l'introduction à son article qu'il envisage de proposer la description d'une analyse de l'interaction en classe de langue étrangère en tant qu'instrument de recherche utilisé par les spécialistes en linguistique appliquée;

(3) cadrage théorique du discours glottodidactique évoqué uniquement dans la bibliographie des articles;

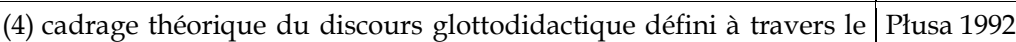
recours aux définitions de notions élaborées dans le champ de la sous-discipline des sciences de l'éducation portant sur la formation des enseignants et que l'on appelle dans le contexte universitaire polonais pedeutologia; toutefois, il faut préciser que P. Płusa (1992) la considère comme une sous-discipline de la glottodidactique et non comme une sous-discipline des sciences de l'éducation, ce qui costitue l'originalité de son approche épistémologique;

(5) cadrage théorique implicite du discours théorique, ce qui constitue Wilczyńska 1998, 1999 - 2 articles; une configuration discursive admise seulement dans le discours des experts dont les publications sont censées être connues par tous les membres d'une communauté de spécialistes ; par exemple, W. Wilczyńska (1998) définit la conception d'un mémoire de diplôme rédigé par les étudiants d'un Collège de Formation des Enseignants de Langues, en s'appuyant sur les modèles de compétence qu'elle a élaborés dans le cadre de son travail de recherche antérieur, sans évoquer ces modèles explicitement;

(6) absence de cadrage théorique dans le discours glottodidactique ; par exemple, M. Kusiak (2004) précise dans l'introduction de son article que celui-ci sera basé sur ses propres réflexions relevant de ses expériences en tant qu'enseignante dans un Collège de Formation des Enseignants de Langues; sans le cadre d'un autre article de cette catégorie, E. Gajewska (2005) se réfère aux données diffusées dans les matériaux informatifs et publicitaires dont les auteurs sont les écoles de langues.
Les articles de Neofilolog

Sawicka 1990; Majewski 1991; Płusa 1993; Niżegorodcew 1994; Zawadzka 1996; Zawadzka 1998; Mrozowska 1998; Wolski 2000; Wiśniewska 2001a; Wiśniewska 2001b; Jankowska 2003; Wysocka 2003; Widła 2003; Adamczak-Krysztofowicz 2004; Zawadzka 2004; Myczko 2004; Niżegorodcew 2004; Wilczyńska 2004; Jaroszewska 2004; Górecka 2004; Strzałka 2004; Gabryś-Barker 2006; Adamczak-Krysztofowicz 2006; Jarząbek 2007; Górecka 2009; Wolski 2010; Orchowska 2010; Karolczuk 2010; Gajewska \& Sowa $2010-29$ articles

Strachanowska 1998; Karpińska-Szaj 2004; Pamuła 2005; Kiliańska-Przybyło 2009; Burlińska 2009; Górska 2009; Witkowska 2010; Stańczyk 2010 (perspective épistémologique propre à la psychologie et/ou aux sciences de l'éducation - 8 article); Majer 2009 (perspective épistémologique de la linguistique -1 article) - 9 articles;

Komorowska 1993; Niżegorodcew 2000 2 articles;

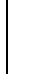

Piasecka 1996; Komorowska 1996; Marciniak 2004; Wiśniewska 1996; P. Płusa 1996; Stasiak 1998; Kusiak 2004; Bandura 2003; S. Wiśniewska 1998; Marciniak 1996; Siek-Piskozub 1996; Frankiewicz 1998; Gajewska 2005; Myczko, Siek-Piskozub 1998; Niżegorodcew 2010; Krakowian 1998; Wysocka 2009 - 17 articles. 
Quand on se penche sur les théories auxquelles se réfèrent les auteurs des articles analysés, on doit reconnaître que parmi les théories scientifiques évoquées se trouvent non seulement les théories construites dans le champ de la glottodidactique mais également celles élaborées dans le champ des sciences connexes. Ceci se justifie par la complexité et le caractère interdisciplinaire de l'objet de recherche en glottodidactique qu'est la formation des enseignants de langues étrangères. Les théories empruntées aux sciences connexes devraient être adaptées à la spécificité épistémologique de la glottodidactique mais à présent certains spécialistes en glottodidactique, tout en se prononçant sur les questions relatives au processus d'enseignement/apprentissage des langues, inscrivent leur réflexion dans la perspective des sciences connexes, notamment les sciences de l'éducation et la psychologie ( 9 articles sur 60 analysés).

Notre méta-analyse montre également que les réflexions sur la formation des enseignants de langue présentées dans les articles publiés dans Neofilolog sont situées épistémologiquement non seulement par rapport à un cadrage théorique mais aussi en référence aux théories dites subjectives (MichońskaStadnik 2013) ou bien aux informations diffusées dans les documents administratifs, éducatifs et dans les recommandations du Ministère de l'Éducation Nationale, du Ministère de l'Enseignement Supérieur et de la Recherche ou du Conseil de l'Europe. On peut relier ceci au caractère empirique de la glottodidactique et le fait que la définition de l'objet de réflexion de la part de l'auteur d'un article scientifique portant sur la formation des enseignants nécessite sa contextualisation. Or, pour reconstituer ce contexte, il faut se référer aux informations pratiques qui n'ont pas toujours une valeur scientifique telles que : les programmes de l'enseignement en vigueur, le nombre des apprenants/étudiants dans le cursus, la politique éducative appliquée actuellement dans le pays, les cultures éducatives, etc.

Et même si au premier abord les données relatives au contexte éducatif semblent dépasser l'objet d'intérêt des spécialistes en glottodidactique, cela ne devrait pas être le cas. En fait, il suffit d'évoquer les modèles du système glottodidactique proposés par les chercheurs polonais à travers l'histoire de la glottodidactique, et notamment celui de Pfeiffer (2001), et celui de Gębal (2013), pour s'apercevoir que parmi les éléments qui composent le système glottodidactique il y a également ceux qui font partie du contexte socioéducatif. Par ailleurs, la contextualisation constitue toujours en glottodidactique le point de départ pour définir l'objet de la recherche à entreprendre, qu' elle soit théorique ou empirique.

De plus, le caractère empirique de la glottodidactique a un impact considérable sur la façon dont les spécialistes en formation des enseignants de 
langues se construisent comme des experts dans leurs discours. Ainsi, à côté des spécialistes qui se prononcent en tant que scientifiques expérimentés, on distingue les spécialistes qui se considèrent comme experts en matière d'enseignement supérieur (Kusiak 2004). Ce deuxième cas reflète toutefois le problème lié à la définition de l'objet d'une réflexion glottodidactique conformément aux critères de la scientificité de cette discipline. Or, sur ce plan, comme nous l'avons déjà mentionné ci-dessus, il reste encore beaucoup de démarches méta-glottodidactiques à entreprendre (Dakowska 2014).

Pour clore la présentation des résultats de notre méta-analyse, nous devons reconnaître que le discours glottodidactique polonais sur la formation des enseignants de langues étrangères est très hétérogène. De plus, cette hétérogénéité reflète bien la spécificité épistémologique de la glottodidactique, et notamment l'absence de modèle unique de savoir glottodidactique et de critères relatifs à sa construction et sa diffusion à travers le discours. Ceci ne facilite pas le dialogue scientifique entre les membres de la communauté glottodidactique polonaise et entraîne, d'une certaine façon, l'absence de liens explicites entre différentes recherches sur la formation des enseignants de langues étrangères menées en Pologne.

\section{EN GUISE DE CONCLUSION}

Nous pouvons conclure de ce qui précède que l'on ne peut pas parler à présent d'une seule vision de la glottodidactique en tant que science et que le discours glottodidactique polonais sur la formation des enseignants de langues reflète parfaitement cet état de choses. Si tous les spécialistes présentent à travers leurs discours la glottodidactique en tant que science empirique, leurs attitudes à l'égard des critères de scientificité de leur domaine de référence varient d'un auteur à l'autre de même que la façon dont ils situent épistémologiquement leurs réflexions et recherches empiriques.

Quant au cadrage théorique, la méta-analyse des articles que nous avons effectuée montre qu'il n'y pas de modèle de référence commun à tous les auteurs en matière de réalisations discursives de ce cadrage. De plus, un nombre considérable d'auteurs des articles sur la formation des enseignants publiés dans Neofilolog ne voit pas la nécessité de donner un cadre théorique explicite à leur discours (19 articles sur 60). Or, une telle absence de cadrage théorique ne nous paraît justifiée que dans le cadre des discours dont les auteurs sont des spécialistes connus de tous les membres de la communauté glottodidactique, ce qui présuppose que leurs publications soient également connues. Les autres auteurs, eux, devraient situer leurs publications dans un cadre théorique, d'autant plus que la glottodidactique est une science plu- 
riparadigmatique et qu'en fonction des théories de référence l'interprétation de l'objet de la réflexion théorique et celle des résultats de la recherche empirique seront différentes.

Pourtant, cette diversité au niveau des pratiques discursives des spécialistes dans la formation des enseignants de langues étrangères rend difficile le dialogue scientifique à l'intérieur de la communauté glottodidactique. En effet, une partie seulement des scripteurs applique le modèle d'écriture de recherche basé sur le dialogisme qui se manifeste à travers leur échange avec les autres spécialistes. Et même si le dialogue conditionne le progrès scientifique, certains auteurs des articles glottodidactiques se limitent à présenter les idées fondées uniquement sur leurs propres expériences, sans prétendre à la rupture épistémologique et sans prendre de distance à l'égard de leurs vécus personnels. D'autres encore se limitent à la définition du contexte empirique dans lequel le problème qu'ils abordent se manifeste, mais en même temps renoncent à conceptualiser et à comprendre ce problème à travers le recours aux savoirs scientifiques qui s'y rapportent. Si les résultats de notre méta-analyse présentés dans la publication antérieure (Orchowska 2015b) ont bien montré la spécificité de la sous-discipline de la glottodidactique qu'est la recherche sur la formation des enseignants de langues étrangères, le présent article illustre surtout l'inscription de ce dernier champ dans l'ensemble des recherches glottodidactiques. En fait, l'analyse des réflexions des spécialistes de formation des enseignants à travers leur discours confirme avant tout que la glottodidactique est une science empirique toujours en train d'établir les fondements de son autonomie interne et externe et que son objet est complexe, voire interdisciplinaire, ce qui pousse les spécialistes à s'intéresser également aux théories construites dans les sciences connexes.

L'état actuel de la glottodidactique en tant que science, ainsi que la spécificité discursive du fonctionnement des membres de la communauté des spécialistes dans l'enseignement/apprentissage des langues étrangères nécessitent non seulement des recherches méta-glottodidactiques (Orchowska 2015a), mais aussi des changements considérables dans la formation des chercheurs dans ce domaine. En fait, la formation de ces derniers devrait inclure le développement de leur compétence discursive en matière de discours glottodidactique, y compris en ce qui concerne la dimension épistémologique de ce discours. Les formateurs, quant à eux, devraient tenir compte également de l'état de la conscience épistémologique de leurs étudiants qui constitue le fondement de leurs connaissances scientifiques et de leur application (Karpińska-Musiał \& Orchowska 2014).

Il est indéniable que les recherches méta-glottodidactiques peuvent s'avérer cruciales pour une meilleure compréhension et pour la qualité re- 
dactionnelle du discours glottodidactique à travers lequel les savoirs sur la formation des enseignants sont construits et diffusés. De plus, cela pourrait par la suite faciliter la communication scientifique à l'intérieur de la communauté glottodidactique, et par conséquent faciliter aussi la coopération scientifique à la fois entre les chercheurs appartenant à cette communauté et également avec les enseignants de langues. Par ailleurs, une telle coopération constitue une condition du progrès scientifique dans le champ de la formation des enseignants de langues étrangères, car ce progrès scientifique pourra ainsi non seulement contribuer à de bonnes pratiques en matière de formation des enseignants mais aussi améliorer tout le processus d'enseignement/apprentissage des langues vivantes.

\section{RÉFÉRENCES}

Boch, F. / Grossmann, F. / Rick, F. (2009). Le cadrage théorique dans l'article scientifique: un lieu propice à la circulation du discours. In: J. M. Lôpez Munoz / S. Marnette/ L. Rosier (ed.), Actes du Colloque Ci-Dit Circulation des discours et liens sociaux: Le discours rapporté comme pratique sociale Québec 2006 (pp. 23-42). Québec: Nota Bene. https://halshs.archi ves-ouvertes.fr/halshs-00600018.

Dakowska, M. (2014). O rozwoju dydaktyki języków obcych jako dyscypliny naukowej. Warszawa: Wydawnictwo UW.

Gębal, E. P. (2013). Modele ksztatcenia nauczycieli JO w Polsce i w Niemczech. W strone glottodydaktyki porównawczej. Kraków: Księgarnia Akademicka.

Jaroszewska, A. (2014). O glottodydaktyce słowami glottodydaktyków. Jezzyki Obce w Szkole, 4, 52-66.

Karpińska-Musiał, B. / Orchowska, I. (2014). Świadomość przedmiotowa i epistemologiczna nauczyciela -refleksyjnego praktyka z perspektywy polskiej glottodydaktyki. Neofilolog, 43/1, 25-38.

Kuhn, T. S. (1962). The Structure of Scientific Revolutions. Chicago: University of Chicago Press.

Lefebvre, M. (2006). Les écrits scientifiques en action. Pluralité des écritures et enjeux mobilisés. Sciences de la Société, 67, 3-15.

Michońska-Stadnik, A. (2013). Teoretyczne i praktyczne podstawy weryfikacji wybranych teorii subiektywnych w kształceniu nauczycieli języków obcych. Wrocław: Wydawnictwo Uniwersytetu Wrocławskiego.

Mondada, L. (1995). La construction discursive des objets de savoir dans l'écriture de la science. Réseaux, 13/71, 55-77.

Niżegorodcew, A. (2009). Foreign language teaching in dialogue. In: Kusiak, M. (ed.), Dialogue in foreign language education (pp. 11-19). Kraków: Jagiellonian University.

Orchowska I. (2013). De la communication spécialisée à l'intérieur de la communauté glottodidactique polonaise. Conceptualisation de l'objet de la discipline scientifique et de son interdisciplinarité. In: E. Wasikiewicz-Firlej / H. Lankiewicz (ed.), From classroom to workplace: advances in applied linguistics (pp. 189-206). Piła: Wydawnictwie PWSZ im. S. Staszica w Pile. 
Orchowska, I. (2015a). De la nécessité des recherches méta-glottodidactiques au sein de la communauté polonaise des spécialistes dans l'enseignement/apprentissage des langues étrangères. Glottodidactica, 42 (2), 169-184.

Orchowska, I. (2015b). O transferencji wiedzy naukowej i subiektywnych poglądów na temat kształcenia nauczycieli języków obcych na podstawie rezultatów metaanalizy artykułów opublikowanych na łamach czasopisma naukowego "Neofilolog". Lingwistyka Stosowana, 12, 81-96.

Pfeiffer, W. (2001). Nauka języków obcych. Od praktyki do praktyki. Poznań: Wagros.

Swales, J. M. (1990). Genre Analysis. English in academic and research settings. Cambridge: CUP.

Wilczyńska W. (2010). Obszary badawcze glottodydaktyki. Neofilolog, 34, 21-35.

Wiśniewska, D. (2013). Action research in EFL pedagogy. Theory and Analysis of Practice. Poznań: Wydawnictwo Naukowe UAM.

\section{ANNEXE \\ - liste des publications qui ont été l'objet de la méta-analyse}

Adamczak-Krysztofowicz, S. (2004). Teksty autentyczne jako źródło interkulturowej kompetencji komunikacyjnej. Neofilolog, 25, 36-42.

Adamczak-Krysztofowicz, S. (2006). Miejsce i rola nauczania interkulturowego na studiach germanistycznych i w kolegiach nauczycielskich w Polsce. Prezentacja wybranych wyników badań empirycznych. Neofilolog, 28, 25-32.

Bandura, E. (2003). Rola nauczyciela języków obcych w rozwijaniu kompetencji interkulturowej uczniów szkół średnich. Neofilolog, 23, 63-69.

Bielicka, M. (2013). Kompetencje nauczyciela języka obcego w przedszkolu immersyjnym. Przygotowanie studentów kierunków neofilologicznych do wykonywania zawodu nauczyciela w przedszkolu dwujęzycznym. Neofilolog, 40/2, 251- 263.

Burlińska, W. (2009). Dyskurs edukacyjny w kształceniu przyszłych nauczycieli. Neofilolog, 33, 169-183.

Frankiewicz, M. (1998). Modele kształcenia nauczycieli języków obcych na przykładzie kształcenia nauczycieli na Uniwersytecie w Ostravie. Neofilolog, 16, 40-44.

Gabryś-Barker, D. (2006). Nauczyciele (jeszcze) nieprofesjonalni: diagnoza problemów (badania kwestionariuszowe). Neofilolog, 29, 90-100.

Gajewska, E. (2005). Kształcenie nauczycieli nie tylko dla szkoły. Neofilolog, 27, 25-30.

Gajewska, E. / Sowa M. (2010). Metodologia konstrukcji kursu języka obcego dla potrzeb zawodowych. Neofilolog, 35, 243-253.

Górecka, J. (2004). Rola nauczającego w procesach konstruowania wiedzy przez studentów kierunków filologicznych podczas seminariów magisterskich. Neofilolog, 25, 59-68.

Górecka, J. (2009). Wprowadzanie elementów nauczania hybrydowego w kształceniu językowym; uzasadnienie dydaktyczne oraz główne wyzwania dla nauczyciela. Neofilolog, 33, 41-52.

Górska, W. (2009). Narzędzie Web 2.0 w codziennej pracy nauczyciela języków obcych. Neofilolog, 33, 197-203.

Jankowska, A. (2003). Wykładowcy w kolegiach językowych wzorem dla przyszłych nauczycieli. Neofilolog, 23, 28-35.

Jaroszewska, A. (2004). Docenić dziecięcy świat - ku nauczaniu zintegrowanemu. Geneza kształcenia zintegrowanego a nauczanie języków obcych dzieci. Neofilolog, 24, 14-23. 
Jarząbek, A. D. (2007). O nastawieniu nauczycieli do samooceny uczących się. Neofilolog, 30, 43-54.

Karolczuk, M. (2010). O wybranych aspektach determinujących nauczanie i uczenie się języka rosyjskiego (drugiego obcego) - wnioski z badań. Neofilolog, 35, 185-194.

Karpińska-Szaj, K. (2004). Integracja dziecka głuchego w klasie językowej. Neofilolog, 24, 33-40.

Kiliańska-Przybyło, G. (2009). Rozwijanie refleksyjności nauczycieli języków obcych na przykładzie techniki analizy zdarzeń krytycznych. Studium przypadku. Neofilolog, 33, 65-75.

Komorowka, H. (1993). Rola i styl kierowania a sukces zawodowy nauczyciela języka obcego. Neofilolog, 6, 7-16.

Komorowska, H. (1996). Kryteria konstrukcji i ewaluacji programów nauczania. Neofilolog, 12, 13-19.

Krakowian, D. (1998). Teaching Foreign Language Methodology - time for modifications. Neofilolog, 16, 22-27.

Kusiak, M. (2004). Problem integracji w organizowaniu praktyk pedagogicznych. Neofilolog, 25, 69-74.

Majer, J. (2009). Analiza dyskursu na lekcji języka obcego. Neofilolog, 32, 99-114.

Majewski, W. (1991). Programowanie neurolingwistyczne w kształceniu nauczycieli języków obcych. Neofilolog, 3, 73-78.

Marciniak, I. (2004). O integracji sprawności językowych w programie kształcenia nauczycieli języka niemieckiego. Neofilolog, 24, 58-62.

Marciniak, M. (1996). Kształcenie i doskonalenie nauczycieli języków obcych w województwie sieradzkim. Neofilolog, 13, 31-34.

Mrozowka, H. (1998). Developing a course in literature for teacher trainees. Neofilolog, 16, 28-33.

Myczko, K. (2004). Koncepcja integracyjna w dydaktyce drugiego języka obcego i praktyka szkolna. Neofilolog, 24, 52-57.

Myczko, K. / Siek-Piskozub T. (1998). Kształcenie absolwentów NJKO na dwuletnich studiach magisterskich w zakresie filologii angielskiej i niemieckiej w Poznaniu. Neofilolog, 16, $54-55$.

Niżegorodcew, A. (1994). Konserwatyzm i innowacja w nauczaniu języków obcych. Neofilolog, $8,20-26$.

Niżegorodcew, A. (2000). Expectations and realities - a teacher training course focused on young learners. Neofilolog, 19, 91-96.

Niżegorodcew, A. (2004). Kształcenie kompetencji socjokulturowej nauczycieli a nauczanie języka angielskiego jako języka międzynarodowej komunikacji. Neofilolog, 24, 63-68.

Niżegorodcew, A. (2010). Europejskie nauczycielskie studia magisterskie (EMETT) - moduły interkulturowe. Neofilolog, 35, 235-241.

Orchowska, I. (2010). Konstruowanie projektu pracy dyplomowej jako element kształcenia akademickiego przyszłych nauczycieli języków obcych. Neofilolog, 34, 111-122.

Pamuła, M. (2005). Boîte à lettres (primary letter box), czyli przygotowanie nauczycieli do nauczania czytania $w$ języku obcym w myśl koncepcji zindywidualizowanego nauczania przy wykorzystaniu technik multimedialnych. Neofilolog, 27, 79-83.

Piasecka, L. (1996), Pre-service teacher training: suggestions for M.A. degree seminars and theses. Neofilolog,12, 36-40.

Płusa, P. (1992). Doskonalenie umiejętności zawodowych nauczyciela języka francuskiego. Neofilolog, 4, 74-77.

Płusa, P. (1993). Warunki efektywnego kształcenia nauczycieli wczesnego nauczania języka francuskiego (na przykładzie kolegium w Sosnowcu). Neofilolog, 6, 25-28. 
Płusa, P. (1996). Walory poznawcze i kształcące czynności w ramach seminarium dyplomowego studentów III roku NKJF. Neofilolog, 13, 57-58.

Sawicka, M. (1990). Zastosowanie magnetowidu w kształceniu nauczycieli języków obcych. Neofilolog, 2, 71-75.

Siek-Piskozub, T. (1996). Rola organizacji nauczycieli języków obcych w doskonaleniu zawodowym nauczycieli. Neofilolog, 13, 64-68.

Stańczyk, J. (2010). Poprzez refleksję do postawy refleksyjnej przyszłego nauczyciela języka obcego - trudności w interpretacji badania w działaniu. Neofilolog, 34, 159-167.

Stasiak, H. (1998). Perspektywy zawodowe absolwentów Nauczycielskich Kolegiów Języków Obcych. Neofilolog, 16, 6-12.

Strachanowska, I. (1998). Foreign Language Teachers - selected problems of self-estimation. Neofilolog, 16, 45-53.

Strzałka, A. (2004). Zintegrowanie nauczania języka i kultury. Neofilolog, 25, 6-9.

Widła, H. (2003). Wykorzystanie wyników opracowań statystycznych w pracy nauczyciela na przykładzie egzaminów gimnazjalnych i próbnych matur. Neofilolog, 23, 88-94.

Wilczyńska, W. (1998). Praca dyplomowa - cele i koncepcje. Neofilolog, 16, 13-21.

Wilczyńska, W. (1999). Kształcenie do autonomii w językach obcych a problem wzorców i imitacji. Neofilolog, 18, 18-24.

Wilczyńska, W. (2004). O mediacji interkulturowej na przykładzie praktyk w zakresie gościnności w Europie. Neofilolog, 24, 69-79.

Wiśniewska, H. (1996). Miejsce czasopisma w kształceniu, dokształcaniu i doskonaleniu nauczycieli języków obcych. Neofilolog,12, 41-45.

Wiśniewska, D. (2001b). Rola nauczyciela w kształtowaniu autonomii ucznia. Neofilolog, 20, 52-57.

Wiśniewska, D. (2001a). Oczekiwania osób uczących się wobec nauczyciela. Neofilolog, 20, 58-63.

Wiśniewska, S. (1998). System studiów dwukierunkowych - propozycja zmian w kształceniu nauczycieli JO. Neofilolog, 16, 34-39.

Witkowska, M. (2010). Zastosowanie protokołu głośnego myślenia i retrospekcji w badaniach autorefleksji przyszłych nauczycieli języka angielskiego. Neofilolog, 34, 147-157.

Wolski, P. (2000). Rola teorii subiektywnych w badaniach autonomii uczenia się języków obcych. Neofilolog, 19, 51-59.

Wolski, P. (2010). Indywidualne teorie na temat nauczania na odległość. Neofilolog, 34, 287-289.

Wysocka, M. (2003). Możliwości badań empirycznych w glottodydaktyce. Neofilolog, 23, 95-100.

Wysocka, M. (2009). Wywiad i obserwacje lekcji w badaniach nad osobą nauczyciela JO. Neofilolog, 32, 139-149.

Zawadzka, E. (1996). Działania innowacyjne a kształcenie nauczycieli. Neofilolog, 13, 23-30.

Zawadzka, E. (1998). Przemiany edukacyjne a kształcenie nauczycieli języków obcych. Neofilo$\log , 17,6-15$.

Zawadzka, E. (2004). Integracja w nauczaniu języków obcych - zarys problematyki. Neofilo$\log , 24,6-13$.

Received: 31.12 .2015 ; revised: 14.05 .2017 